\title{
HABILIDADES INVESTIGATIVAS EN LA COMPRODUCCIÓN LECTORA VIRTUAL
}

\author{
INVESTIGATIVE SKILLS IN THE VIRTUAL READING COMPREHENSION
}

\author{
Waldemar José Cerrón Rojas ${ }^{1}$
}

\section{RESUMEN}

Se proyectan conexiones entre habilidades investigativas y la comproducción lectora virtual. Conexión ideoactiva necesaria entre autocontrol, flexibilidad, precisión, solidez, transferencia, economía, simulación y realidización transdisciplinaria para revitalizar (neo) (re) conceptos, categorías, estructuras o teorías. Soporte necesario de la transproducción conectiva de matrices epistémicas, conocimientos, y proyecciones estéticas orientada a superar limitaciones históricas emergentes.

Palabras clave: Conexión ideoactiva, comproducción lectora virtual, habilidades investigativas, transproducción.

\section{ABSTRACT}

Connections are projected between investigative skills and virtual reading performance. Ideoactive connection between selfcontrol, flexibility, precision, solidity, transference, economy, simulation and transdisciplinary realignment to revitalize (re) concepts, categories, structures or theories. Necessary support for the connective transduction of epistemic matrixes, knowledge, and aesthetic projections aimed at overcoming emerging historical limitations.

Keywords: Ideoactive connection, virtual reader verification, investigative skills, transproduction.

\section{INTRODUCCIÓN}

La conexión de las habilidades investigativas conectadas con la comproducción lectora virtual soporta la configuración del sentido emergente histórico - lógico de neo conocimientos.

Las habilidades investigativas son ideoactividades (idea y actividad) conscientes que activan sentimientos, pensamientos, imaginarios, conocimientos, decisiones, voluntades, acciones previas, recurrentes y posteriores. Vinculadas a la comprensión lectora virtual conectan sistemática y simultáneamente conocimientos digitalizados con (re) (neo) conceptualizaciones, categorizaciones, estructuraciones que configurarán neo teorías emergentes. Lo que significa transparentar la superación de limitaciones históricas.

Durante la comproducción lectora virtual las habilidades investigativas visibilizan conocimientos emergentes. De esta manera el autocontrol faculta la utilización de herramientas múltiples; la flexibilidad proporciona alternativas viables; la solidez soporta ideológicamente los argumentos, la economía significa control operativo, la precisión gestión metodológica; la operacionalización transmutación de las estructuras a diversos contextos; la virtualización proyección de la propuesta emergente y la realidización la transferencia a la realidad.

Los conocimientos digitales emergentes pueden adquirir la forma de concepto (determinante, atributo, complemento) o categorías (identificación, selección, codificación) o estructuras (jerarquía, relación, sistema) o teorías (limitaciones, históricas, matriz epistémica, teoría y propuesta estética emergente) que describen, explican, interpretan la realidad. Soportadas en la práctica ideológica rigurosa, sistemática, crítica, emancipatoria de las habilidades investigativas.

La comproducción lectora virtual es la transfase consciente, científica y artística del virtual nauta con la simulación contenida, pudiendo ser compresora 0 productora. Se capta el sentido, significado propuesto en la transfase comprensora proyectando la posibilidad de organizar las conexiones internas y relaciones externas de la realidad virtualizada. Esta interacción es de 
carácter transdisciplinario porque convoca la presencia de hipertextos, comunidades de aprendizaje e hipermundos.

Las habilidades investigativas soportan la comproducción lectora virtual. Conexión necesaria para la emergencia de la transproducción (transferencia y producción) de nuevos conocimientos. El uso y dominio consciente de leyes, categorías y herramientas determinará el significado científico de lo comproducido; mientras que las propuestas emergentes denotarán el sentido social de su efecto estético. Transtextualizar las limitaciones históricas constituye un acercamiento a la felicidad de los pueblos.

\section{Habilidades investigativas en la comproducción lectora virtual}

Las habilidades investigativas constituyen el soporte fundamental para la producción y transferencia de conocimientos en la comproducción lectora virtual. Fundamentalmente cuando se pretende (re) (neo) conceptualizaciones, categorizaciones, estructuraciones y teorías emergentes no convencionales hasta que sean conocidas. Dichas ideoactividades son necesarias para incrementar el conocimiento digital primigenio, el cual realidizado soportará los avances culturales locales yglobales.

Remarcan su sentido investigativo cuando se logra transtextualizar, virtualizar, simular, transfasar el sentido y significado propuesto en lectura digital. Cerrón y Rojas (2015) concluyen que las habilidades investigativas constituyen actividades interdisciplinarias de actuación concreta. Los comproductores virtuales al aplicarlas soportarán científicamente la utilización de técnicas, prácticas, métodos, herramientas necesarias para cumplir objetivos, de acuerdo a la naturaleza y características particulares de la lectura digital.

El dominio de las habilidades investigativas significa el despliegue consciente de la comproducción lectora virtual. Visualizan, simulan y realidizan el sentido y significado de la virtualización de conceptos, categorías, estructuras y teorías propuestas. Dinamizan la determinación gradual de procedimientos necesarios para virtualizar el sentido ideológico del texto digital, transparentar el significado de sus limitaciones históricas, simulación de conceptos, categorías, estructuras, teorías y propuesta emergente de su efecto estético.

Las habilidades investigativas facultan a los comproductores la mejora continua de la comprensión lectora y producción de textos virtuales. Al vincularlas con actividades y acciones de simulación, construcción y reconstrucción de mundos virtuales paralelos refuer- za la interacción ideológica (sentimientos, pensamientos, conocimientos, deseos, voluntad y acción) para (re) conceptuar, categorizar, estructurar y teorizar las ciencias económicas, administrativas y contables.

Entonces las habilidades investigativas son cualidades que permiten la interacción dinámica metodológica científica del comproductor con la simulación y realidización. Se movilizan en dos transfases; comprensora y comproductora. En la primera se visibiliza el sentido del conocimiento en la matriz epistémica; en la segunda se conecta con regularidades investigativas, leyes y categorías socio ambientales para la producción, simulación de conocimientos, así como el efecto artístico de la propuesta para alcanzar ideales humanamente bellos.

\section{Transfase comprensora}

Se conecta con las habilidades de autocontrol, economía, precisión y solidez para interactuar con el sentido, significado de conocimientos virtualizados. Se transfasa el sentido histórico dinamizador de formas de hacer y conocer utilizando herramientas de la red (resaltado, subrayado, diccionarios, blogs, aulas virtuales, videos, dobles virtuales) que hipervinculan información y evidencian lo comprendido. Visibiliza la forma de hacer y conocer de la una cultura, los límites de su conocimiento y efecto estético propuesto (para qué escribió).

\section{Transfase comproductora}

Establece conectividad con las habilidades de flexibilidad, solidez, precisión, transferencia y realidización. El virtual nauta aplica trasndisciplinariamente regularidades investigativas, leyes, categorías y herramientas digitales para simular, producir y realidizar estéticamente nuevos conceptos, categorías, estructuras y teorías.

Conecta este mundo con otros posibles mediante la simulación, actividad que abrevia procesos y aliviana el peso histórico de las deformidades sociales en la dinámica socio ambiental productiva.

\section{Regularidades investigativas}

La ley es la conexión interna, necesaria, esencial entre fenómenos manifiesta a través de regularidades generales y aplicadas de manera particular. Las categorías son la síntesis del alcance del conocimiento en la historia, cuyo desarrollo se visibiliza mediante conceptos, juicios y razonamientos. El conocimiento de estas regularidades y categorías asociadas a las habilidades investigativas es el punto de partida para la comproducción lectora virtual.

El conocimiento es la conexión necesaria del compro- 
ductor hacia el movimiento interno, elemento dinamizador, conexiones esenciales, propiedades universales de los temas abordados en el texto digital. Ambas transfases comproductoras son posibles mediante la interconectividad de conceptualización, categorización, estructuración, contrastación y teorización respectiva. Estas conexiones casuales hacen que necesariamente se (re) conceptualice, categorice, estructure, contraste y teorice transtextualmente.

La conceptualización es el sentido histórico ideológico para la denominación correspondiente a objetos, fenómenos que pueden revelar el carácter, causas subyacentes para entender sus conexiones internas y relaciones externas. De manera que en la categorización se organizan los conceptos en clases significativas de acuerdo a sus conexiones respectivas; luego de un proceso de identificación, selección y codificación ubicará los conceptos que determinarán la nueva estructura del conocimiento.

La estructuración comprende la conexión de las categorías correspondientes a una secuencia de jerarquización, relación y sistematización. El comproductor conceptúa, categoriza, estructura, sistematiza la propuesta teórica. En consecuencia la teorización es una herramienta descriptiva, explicativa, interpretativa para entender y actuar respecto de la propuesta de conocimiento en el texto digital. De esta manera se va incrementado la transproducción permanente de conocimientos auténticos.

Al respecto Rosental y Stracks ( 1960) refieren de las categorías del materialismo dialéctico. Fenómeno y esencia expresan la unidad indisoluble de las conexiones internas y relaciones externas de los temas abordados en el texto. El acceso a la esencia es posible mediante el pensamiento abstracto; mientras el fenómeno permanece visible. Causa y efecto conectan nexos causales, estables repetitivos y necesarios en el desarrollo histórico de objetos o fenómenos, de tal manera que su aplicación permite el descubrimiento de sus regularidades.

Necesidad y casualidad están conectadas porque históricamente la necesidad soporta la casualidad, en la que se halla la ley; entonces el comproductor deberá transformar la casualidad en necesidad. En el contenido y la forma se manifiestan movimientos internos y su estructurada es la forma en que se presentan en la historia. Lo singular, particulary universal se expresa mediante en tanto que lo particular concretiza lo universal como lo universal incluye a lo particular.

La realidad y posibilidad se interconectan en lo que existe, cuya simulación necesariamente es posible de existir. Asíla posibilidad simulada puede realidizarse de acuerdo a las condición socio ambiental. Lo histórico y lógico soportan lo real y la representación de su orden, permite interconectar leyes, categorías, establecer identidad, comprensión y explicación suficientes para dinamizar el desarrollo. Lo concreto posibilita la interacción sensible del objeto; mientras lo abstracto con su contenido y esencia, aspectos contradictorios; pero generalizables.

Esta base faculta la ideoactivación de conexiones, proyecciones, construcciones necesarias para realidizar la comproducción lectora virtual. Las habilidades investigativas autocontrol (instrumento), flexibilidad (alternativas) transferencia (operacionalización), precisión (métodos), solidez (argumentación), economía (tiempo) simulación (propuesta), virtualización (entorno virtual) realidización (transferencia a la realidad) revitalizan constantemente transproducción de conocimientos a partir de lo propuesto en la lectura digital.

\section{Habilidades investigativas}

\section{Autocontrol}

Secuencia de ideoactividades que de manera lógica conectan la comproducción lectora virtual con la aplicación de instrumentos concretos para verificar sus procesos inherentes. La virtualización, simulación y realidización deberán ser ejecutados con el acompañamiento de instrumentos válidos y confiables. La ficha de verificación o cualquier otro instrumento que sirva de guía concreta, durante el desarrollo abstracto de la comproducción lectora. 
Tabla 1. Lista de verificación de las habilidades investigativas

\begin{tabular}{|c|c|c|c|}
\hline Habilidades & Indicador & sí & no \\
\hline Precisión & $\begin{array}{l}\text { Evidencia perfeccionamiento del dominio metodológico durante la } \\
\text { comproducción lectora virtual. }\end{array}$ & & \\
\hline Economía & $\begin{array}{l}\text { Perfeccionan el cumplimiento operativo de las etapas pre establecidas durante la } \\
\text { comproducción lectora virtual. }\end{array}$ & & \\
\hline Transferencia & $\begin{array}{l}\text { Perfecciona la operacionalización con efectividad el desarrollo de la durante la } \\
\text { comproducción lectora virtual. }\end{array}$ & & \\
\hline Flexibilidad & $\begin{array}{l}\text { Perfecciona la propuesta de alternativas viables y significativas durante la } \\
\text { comproducción lectora virtual. }\end{array}$ & & \\
\hline Solidez & $\begin{array}{l}\text { Perfecciona la interiorización y exteriorización esencial de las condiciones } \\
\text { cambiantes durante la comproducción lectora virtual. }\end{array}$ & & \\
\hline Autocontrol & $\begin{array}{l}\text { Perfecciona el control del desarrollo de los procesos de la investigación } \\
\text { científica con instrumentos válidos y confiables durante la comproducción } \\
\text { lectora virtual. }\end{array}$ & & \\
\hline Simulación & $\begin{array}{l}\text { Simula propuestas del conocimiento en el ordenador a fin de ser proyectado en } \\
\text { escenarios futurísticos. }\end{array}$ & & \\
\hline Virtualización & $\begin{array}{l}\text { Virtualiza determinada propuesta para su respectiva transferencia a otros } \\
\text { escenarios o realidades }\end{array}$ & & \\
\hline Realidización & $\begin{array}{l}\text { Transfiere a escenarios reales la virtualización de la simulación mediante su } \\
\text { concretización o producción }\end{array}$ & & \\
\hline
\end{tabular}

\section{Economía}

Ideoactividad que perfecciona el cumplimiento de etapas pre establecidas en el desarrollo de la comproducción lectora. Sistematiza, conecta, ejecuta simultáneamente el control del tiempo establecido; el virtual nauta considerará la distribución creativa, abstracta del sistema operativo concreto, necesarios para la comprensión y producción de los nuevos conocimientos.

\section{Precisión}

Ideoactividad (idea y actividad) sitemática y simultánea que conecta relaciones causales y necesarias de los métodos que se aplicarán durante la comproducción lectora. Destaca la necesidad de comprender y conectar de manera sólida y precisa el conocimiento procedimental para transformar la realidad. La precisión metodológica radica en el dominio de los procedimientos corespondientes, necesarios y causales de método.

\section{Tabla 2. Lista de métodos y procedimientos}

\begin{tabular}{|l|l|}
\hline Métodos & Procedimientos \\
\hline 0bservación & $\begin{array}{l}\text { Selección de objetos, fenómenos o sujetos /elección de muestra representativa de aspectos, eventos o } \\
\text { conductas /definición de unidades de observación/ sistematización de categorías y subcategorías del } \\
\text { objeto u hecho observado y construcción del significado en el conocimiento. }\end{array}$ \\
\hline Medición & $\begin{array}{l}\text { Selección de objetos, fenómenos o sujetos a cuantificarse/ operacionalización del objeto de } \\
\text { estudio/medición de indicadores (nominal, ordinal, intervalo, proporción) con escalas } \\
\text { correspondientes/selección o construcción del instrumento/cuantificación de datos del objeto de } \\
\text { estudio/establecer procedimientos de codificación y conteo/ interpretación de datos } \\
\text { cuantificados/construcción de significados cuantitativos para el conocimiento. }\end{array}$ \\
\hline Experimental & $\begin{array}{l}\text { Delimitación del experimento/condicionamiento artificial de objetos a experimentarse/ejecución del } \\
\text { experimento/observación y medición de datos obtenidos en la experimentación/interpretación de los } \\
\text { resultados obtenidos/inserción del producto al conocimiento }\end{array}$ \\
\hline Descriptivo & $\begin{array}{l}\text { Elegir el objeto o fenómeno a describirse/Caracterizar las partes y el todo del objeto a } \\
\text { describirse/Conceptuar el objeto/Ingresar determinado concepto al conocimiento. }\end{array}$ \\
\hline Explicativo & $\begin{array}{l}\text { Elegir el objeto o fenómeno a explicar/establecer relaciones internas y externas/conceptuar el } \\
\text { objeto/determinar propiedades y cualidades del objeto/definición del objeto de estudio//transferencia } \\
\text { lógica de propiedades y cualidades del objeto de estudio/relación multidisciplinaria del } \\
\text { objeto/evaluación de resultados de la transferencia/ingresar determinada definición al conocimiento. }\end{array}$ \\
\hline
\end{tabular}




\begin{tabular}{|c|c|}
\hline Correlacional & $\begin{array}{l}\text { Organizar la operacionalización comparativa/determinar las relaciones análogas de los objetos o } \\
\text { fenómenos/medir el nivel de fuerzas de los objetos/establecer el nivel de influencia de un objeto sobre } \\
\text { otro/explicar las relaciones del objeto o fenómeno/insertar las comparaciones al conocimiento. }\end{array}$ \\
\hline Modelación & $\begin{array}{l}\text { Selección del objeto a modelar/planificación para la representación de la modelación/construcción del } \\
\text { prototipo del objeto a investigar/simulación real o virtual del prototipo semejante o } \\
\text { diferente/aplicación del prototipo en situaciones concretas/inserción del modelo al conocimiento. }\end{array}$ \\
\hline $\begin{array}{l}\text { Inductivo- } \\
\text { deductivo }\end{array}$ & $\begin{array}{l}\text { Selección del objeto de estudio a inducir o deducir/identificación de propiedades generales y/o } \\
\text { particulares/particularizar y/o generalizar los efectos de las relaciones internas o } \\
\text { externas/establecimiento de normas o leyes de su existencia/regularización de propiedades generales } \\
\text { y/o particulares/aplicación en el conocimiento. }\end{array}$ \\
\hline $\begin{array}{l}\text { Análisis- } \\
\text { síntesis. }\end{array}$ & $\begin{array}{l}\text { Establecer la unidad del objeto de estudio/descomponer el todo en sus partes/clasificar los } \\
\text { componentes generales y particulares/describir las partes externas y las relaciones internas/explicar las } \\
\text { propiedades y cualidades de las partes/identificar la esencia del objeto de estudio/experimentar su } \\
\text { funcionamiento o sistema/integrar las partes para conformar la unidad/establecer la identidad del } \\
\text { objeto de estudio. }\end{array}$ \\
\hline $\begin{array}{l}\text { Abstracto- } \\
\text { concreto }\end{array}$ & $\begin{array}{l}\text { Seleccionar determinada parte del objeto de estudio/transferir al pensamiento la parte } \\
\text { seleccionada/sistematizar sus cualidades y propiedades/transferir a la práctica las cualidades y } \\
\text { propiedades en su movimiento/conceptualizar el objeto seleccionado en su integralidad/aplicar el } \\
\text { concepto en el movimiento y desarrollo real o virtual del conocimiento. }\end{array}$ \\
\hline $\begin{array}{l}\text { Histórico } \\
\text { lógico }\end{array}$ & $\begin{array}{l}\text { Ubicación del objeto de estudio en el tiempo/desen volvimiento del objeto de estudio en el } \\
\text { tiempo/localización de fuentes referenciales/explicación de relaciones internas y } \\
\text { externas/sistematización de causas y efectos/transferencia de reconstrucción lógica/contemporización } \\
\text { del objeto de estudio. }\end{array}$ \\
\hline Genético & $\begin{array}{l}\text { Identificar la unidad genética/caracterizar cadenas estructurales/proponer cambios en el orden de las } \\
\text { estructuras del gen/implantar el gen con las características deseadas en la estructura interna de la } \\
\text { unidad/evaluar propiedades y características de la unidad modificada después de la manipulación en } \\
\text { condiciones controladas/replicar la unidad a gran escala. }\end{array}$ \\
\hline Dialéctico & $\begin{array}{l}\text { Identificación del objeto o fenómeno/seleccionar leyes y propiedades/clasificar sus contradicciones } \\
\text { mediante las categorías (causa/efecto;esencia/fenómeno; realidad/posibilidad; necesidad/ casualidad; } \\
\text { necesidad/libertad) aplicación de leyes y propiedades (contradicción, cambios cuanti cualitativos y } \\
\text { negación de la negación)/explicar contradicción principal y coexistencia de las leyes/manejar el } \\
\text { equilibrio dinámico/garantizar el desarrollo a partir de la contradicción. }\end{array}$ \\
\hline
\end{tabular}

\section{Transferencia}

Las habilidades investigativas de transferencia proyectan en el comproductor la operacionalización efectiva del contenido de los conceptos, categorías, estructuras y teorías para que sean transferidos a otra forma y en otros contextos. De esta manera vinculará permanentemente las bases teóricas a la simulación que quiere realidizar para (re) conceptuar, categorizar, estructurar y teorizar. En este caso se transfieren los procedimientos metodológicos a la comproducción lectora virtual.

\section{Tabla 3. Lista de métodos y procedimientos para la comproducción}

\begin{tabular}{|l|l|}
\hline Métodos & Procedimientos \\
\hline Observación & $\begin{array}{l}\text { Selección del texto digital/elección de conceptos, categorías, estructuras o teorías/definición de } \\
\text { unidades de comproducción / sistematización de (re) conceptos, categorías, estructuras o teorías y } \\
\text { conexión del sentido y significado en la realidad emergente. }\end{array}$ \\
\hline Medición & $\begin{array}{l}\text { Selección del texto digital a cuantificarse/ virtualización de la operacionalización del objeto de } \\
\text { estudio/simulación de la medición de indicadores (nominal, ordinal, intervalo, proporción) con escalas } \\
\text { correspondientes/selección o construcción del instrumento/cuantificación de datos del objeto de } \\
\text { estudio/interpretación de datos cuantificados/realidización de significados cuantitativos para el } \\
\text { conocimiento. }\end{array}$ \\
\hline Experimental & $\begin{array}{l}\text { Delimitación del experimento digital/simulación artificial de objetos a experimentarse/ejecución del } \\
\text { experimento/observación y medición de datos obtenidos en la experimentación/interpretación de los } \\
\text { resultados obtenidos/realidización de experimento. }\end{array}$ \\
\hline
\end{tabular}




\begin{tabular}{|c|c|}
\hline Descriptivo & $\begin{array}{l}\text { Elegir el texto digital a describirse/simular las características de las partes y el todo del objeto a } \\
\text { describirse/Conceptuar el objeto/ realidizar determinado conepto al conocimiento. }\end{array}$ \\
\hline Explicativo & $\begin{array}{l}\text { Elegir el texto digital a explicar/simular las relaciones internas y externas/conceptuar el } \\
\text { objeto/determinar propiedades y cualidades del objeto/definición del objeto de estudio//simulación de } \\
\text { la transferencia lógica de propiedades y cualidades del objeto de estudio/relación multidisciplinaria del } \\
\text { objeto/evaluación de resultados de la transferencia/realidizar determinado argumento al } \\
\text { conocimiento. }\end{array}$ \\
\hline Correlacional & $\begin{array}{l}\text { Organizar la operacionalización comparativa de los textos digitales/virtualiza las relaciones análogas de } \\
\text { los objetos o fenómenos/medir el nivel de fuerzas de los objetos/simula el nivel de influencia de un } \\
\text { objeto sobre otro/explicar las relaciones del objeto o fenómeno/realidiza las comparaciones al } \\
\text { conocimiento. }\end{array}$ \\
\hline Modelación & $\begin{array}{l}\text { Selección del texto digital a modelar/construcción del prototipo del objeto a investigar/simulación real } \\
0 \text { virtual del prototipo semejante o diferente/realidización del prototipo en situaciones } \\
\text { concretas/inserción del modelo al conocimiento. }\end{array}$ \\
\hline $\begin{array}{l}\text { Inductivo- } \\
\text { deductivo }\end{array}$ & $\begin{array}{l}\text { Selección del texto digital a inducir o deducir/identificación de propiedades generales y/o } \\
\text { particulares/particularizar y/o generalizar los efectos de las relaciones internas o externas/virtualizar las } \\
\text { normas o leyes de su existencia/simular sus propiedades general es y/o particulares/realidizarlo en el } \\
\text { conocimiento. }\end{array}$ \\
\hline $\begin{array}{l}\text { Análisis- } \\
\text { síntesis. }\end{array}$ & $\begin{array}{l}\text { Establecer el texto digitial/virtualizar la descomposición en sus partes/clasificar los componentes } \\
\text { generales y particulares/describir las partes externas y las relaciones internas/explicar las propiedades y } \\
\text { cualidades de las partes/simular la esencia del objeto de estudio/realidizar su funcionamiento o } \\
\text { sistema/integrar las partes para conformar la unidad/establecer la identidad estética emergente del } \\
\text { objeto de estudio. }\end{array}$ \\
\hline $\begin{array}{l}\text { Abstracto- } \\
\text { concreto }\end{array}$ & $\begin{array}{l}\text { Seleccionar determinada parte del texto digital/transferir al pensamiento la parte } \\
\text { seleccionada/sistematizar sus cualidades y propiedades/simular el movimiento de cualidades y } \\
\text { propiedades/conceptualizar el objeto seleccionado en su integralidad/realidizar el concepto en el } \\
\text { movimiento y desarrollo real o virtual del conocimiento. }\end{array}$ \\
\hline $\begin{array}{l}\text { Histórico } \\
\text { lógico }\end{array}$ & $\begin{array}{l}\text { Ubicación del texto digital en el tiempo/virtualización del objeto de estudio en el tiempo/localización de } \\
\text { fuentes referenciales/explicación de relaciones internas y externas/sistematización de causas y efectos/ } \\
\text { simulación de la reconstrucción lógica/ realidización de la contemporización del objeto de estudio. }\end{array}$ \\
\hline Genético & $\begin{array}{l}\text { Identificar la unidad genética del texto digital/simular las cadenas estructurales/proponer cambios en el } \\
\text { orden de las estructuras del gen/ realidizar el gen con las características deseadas en la estructura } \\
\text { interna de la unidad/evaluar propiedades y características de la unidad modificada después de la } \\
\text { manipulación en condiciones controladas/replicar la unidad a gran escala. }\end{array}$ \\
\hline Dialéctico & $\begin{array}{l}\text { Identificación del texto digital/virtualizar sus leyes y propiedades/simular contradicciones mediante sus } \\
\text { leyes (contradicción, cambios cuanticualitativos y negación de la negación) y categorías (causa/efecto; } \\
\text { esencia/fenómeno; realidad/posibilidad; necesidad/ casualidad; necesidad/libertad) /realidizar la } \\
\text { contradicción principal para el equilibrio dinámico de la propuesta emergente. }\end{array}$ \\
\hline
\end{tabular}

\section{Flexibilidad}

Habilidad investigativa conectada con las categorías de lo histórico lógico. Tanto las propuestas universales contenidas en el texto digital como las que se van comproduciendo estuvieron y están sujetas históricamente a la viabilidad lógica para la superación particular de las limitaciones históricas, reconstrucción de matrices epistémicas, teorías y propuesta emergentes. Orienta a los comproductores durante la simulación de propuestas en escenarios posibles y futurísticos de realidización.

\section{Solidez}

La habilidad investigativa de la solidez constituye el soporte invisible y fundamental para la comproducción lectora. Perfecciona la simulación argumentativa esencial de condiciones y situaciones emergentes prove- nientes de la lectura digital soportada en leyes, categorías científicas. De esta manera, serán simuladas y comprendidas con mayor precisión la realidización de esencia de las teorías para que sean aceptadas como tal, sin atender solamente el fenómeno.

\section{Simulación}

Es una habilidad que trasluce la propuesta emergente de la comproducción lectora. Son actividades que pueden realizarse dentro del ordenador; representaciones, artificios, imitaciones de situaciones que aún no se han dado en la realidad. Tiene la finalidad de materializar la propuesta estética, se conecta con las categorías de realidad y posibilidad, pues la base de lo real implica la simulación de muchas posibilidades. 


\section{Virtualización}

Ideoactividad que a través del entorno virtual faculta la creación de escenarios, propuestas en un plano más cercano a la realidad. Se conectan diversas posibilidades, las cuales pueden ser visualizadas sin necesidad de su existencia física. Muchos conocimientos pueden ser virtualizados a fin de que no puedan afectar en la realidad, sin embargo su proyección es necesaria, de manera tal, que su realidización no cause efectos negativos 0 pueda ser controlada.

\section{Realidización}

Habilidad necesaria de la comproducción lectora. Es la transferencia de la simulación y virtualización respectiva a la realidad física de manera que se pueda transproducir los conocimientos emergentes de la lectura digital. Soporta la independencia o descolonización del conocimiento debido a que los (neo) (re) conceptos, categorías, estructuras, contrates, teorías adquieren caracteres originales, auténticos y necesariamente complementan lo faltante en el contexto local y global.

\section{CONCLUSIÓN}

Las habilidades investigativas dinamizan la comproducción lectora virtual soportada en el uso, dominio de leyes y categorías científicas. La generación de virtual nautas requiere de esta ideoactividad para transproducir (re) conceptos, categorías, estructuras y teorías cuanti cualitativamente superiores. Su aplicación en las transfaces comprensora y productora transmuta el sentido y significado de la realidad al momento de virtualizar, simular para realidizar la configuración emergente de la propuesta estética.

\section{REFERENCIAS BIBLIOGRÁFICAS}

Cerrón, W. y López, B. (2015). Habilidades investigativas y formación de valores pedagógicos ambientales. Huancayo : UNCP.

Rosental , M., y Stracks, G. (1960). Categorías del materialismo dialéctico . México: Grijalbo .

\section{GLOSARIO}

- Visualizar. Captar transdisciplinariamente la interfaz en el espacio virtual

- Simular. Actividades que puedan realizarse dentro del ordenador

- Realitivización. Acción de transferir lo virtual a lo real

- Transfase. Obligada correspondencia del sujeto con la realidad virtual (visualización, simulación y realitivización)

- Comproducción. Acción de comprender y producir de manera inmediata

- Limitación histórica. Punto de llegada de los acciones del hombre dado el conocimiento de la época.

- Efecto estético. Es la atracción emocional y racional de los sentimientos hacia el producto artístico capaz de desarrollar sentimientos y pensamientos elevados.

- Virtualnauta. Ente que transfaza la comproducción (en este caso la lectora virtual) 